\title{
The Difference of the Theoretical Approach of Corporate Social Responsibility Between the European Union and the United States of America
}

\section{Wahiba Mokadem}

Faculty of Economic, Commercial, Financial and Management Sciences,

University of Abdelhamid Ben Badis,

Mostaganem, Algeria.

Email: wahiba.mokadem@yahoo.com

Bishr Muhamed Muwafak (Orcid 0000-0005-1085-1525)

Applied Science University, Sitra, Bahrain.

Islamic Economics and Finance Pedia.

Email: bishrmm@gmail.com - Bishr.mohd@asu.edu.bh

Received October, 2020; Accepted December, 2020

\begin{abstract}
The experiences of countries that relate to corporate social responsibility differ greatly, and the reason for these differences is due to several reasons, including: the economic, social, and cultural policies of the country, the degree of government intervention in corporate activity, and the degree to which governments cover societal needs. Therefore, we find a difference between the theoretical approaches to social responsibility in both European countries and the USA. This research aims to explain these two approaches, by looking at each approach in an analytical manner and then deducing the differences between them, and this is to give an overview of the advantages and disadvantages of each approach and highlight their significant contributions.
\end{abstract}

Keywords: Corporate Social Responsibility (CSR); Implicit Corporate Social Responsibility; Explicit Corporate Social Responsibility; The American Approach, The European Approach.

Type: Research paper

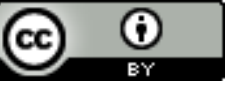

DOI: 10.51325/ijbeg.v4i1.22

الفرق بين المقاربات النظربـة للمسؤولية الاجتماعية للشركات بين كل من دول الاتحاد الأوربي و الولايات المتحدة الأمريكية

ملخص البحث :

تختلف تجارب الدول في مجال المسؤولية الاجتماعية للشركات بشكل كبير، و سبب هذه الاختلافات يعود لعدة أسباب منها: السياسات الاقتصادية و الاجتماعية و الثقافية للدولة و درجة التتخل الحكومي في نثاط الثركات، و درجة تغطية الحكومات للمتطلبات المجتمعية، لذلك نجد اختلافا بين المقاربات النظرية للمسؤولية الاجتماعية في كل من الدول الأوروبية و الولايات المتحدة الأمريكية. يهدف هذا البحث دئ دئه إلى شرح هاتين المقاربتين، من خلال التعرض لكل مقاربة بشكل تحليلي و مفصل و من ثم استنتاج أوجه الاختلاف بينهما، و يتيح ذلك التعرف على مزايا و عيوب كل تجربة و استفادة من التجربتين معا نظرا لأهميتهما.

الكلمات المفتاحية: المسؤولية الاجتماعية للشركات، المسؤولية الاجتماعية الضمنية، المسؤولية الاجتماعية العلنية، المقاربة الأمريكية، المقاربة الأوروبية. 
تسعى الشركة التي تمارس المسؤولية الاجتماعية للشركات إلى أن يكون لها تأثير إيجابي على المجتمع ، والبيئة و

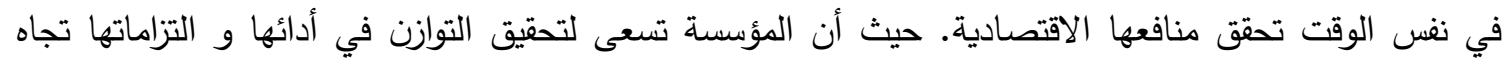

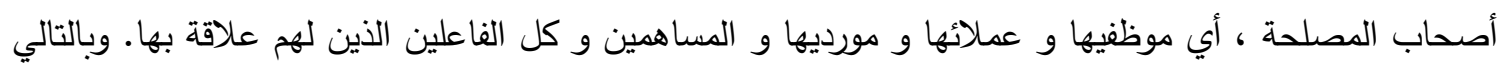
فإن الشركات تتعهد بإدماجها لبرامج المسؤولية الاجتماعية على أساس طوعي و و خارج أي إطار قانوني ملزم.

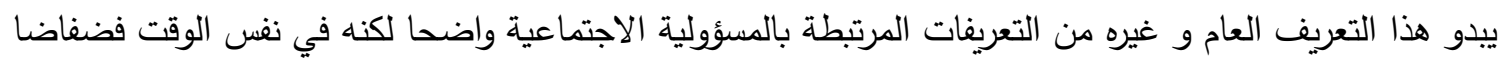

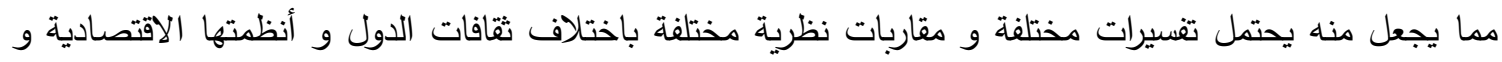
توجهاتها الاجتماعية و حتى الدينية. إذن يمكن لأي دولة أن تكيف مفهوم المسؤولية الاجتماعية لشركاتها بما يتناسب مع خصوصيتها و مبادئها و أهدافها. من هذا المنطلق، فإن هناك العديد من التجارب الدولية في مجال المسؤولية الاجتماعية للشركات، و لكل تجربة

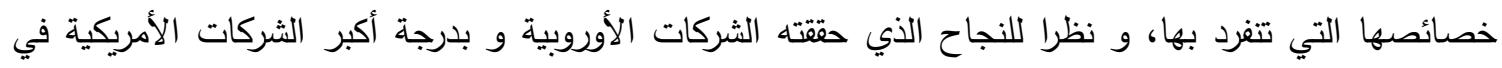
مجال المسؤولية الاجتماعية ، فإننا في هذه الدراسة سنلقي الضوء على هاتين التجربتين بثيء من التحليل من أجل

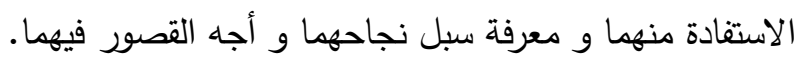

2.1

التعرض للظاهرة البحثية المدروسة كان بالتركيز على تساؤل أساسي هو : ما هو الفرق بين المقاربات النظرية

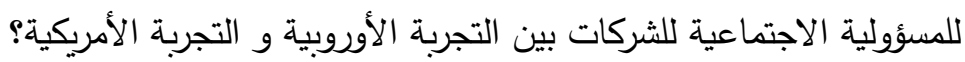

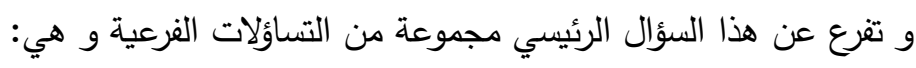

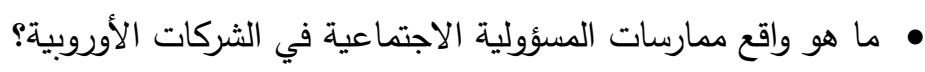

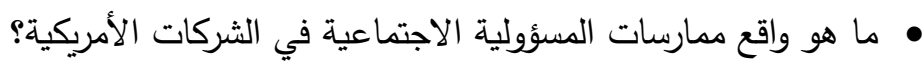
• ما هي أهم الاختلافات بين التجربتين الأمريكية و الأوروبية ؟

3.1

تعتبر التجربتين الأمريكية و الأوروبية رائتين في مجال المسؤولية الاجتماعية للشركات، و لكن لكل تجربة

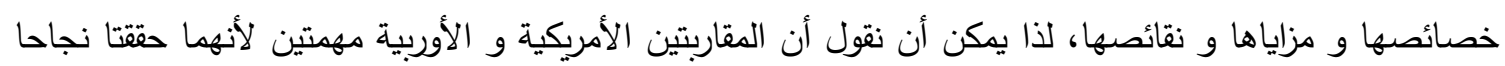

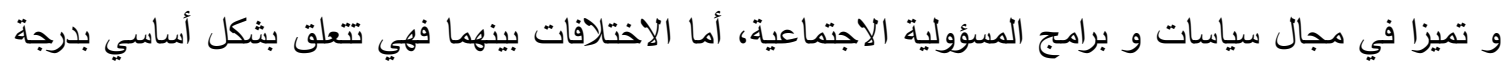
الحرية الممنوحة للشركات في اختيار و ممارسة البرامج المسؤولة.

$$
4.1
$$

تكمن أهمية هذا البحث فيما يلي:

توضيح واقع ممارسة المسؤولية الاجتماعية للشركات في كل من دول الاتحاد الأوربي و الولايات المتحدة الأمريكية من خلال عرض تجاربهما في هذا المجال دراسة المقاربة النظرية للمسؤولية الاجتماعية في كل دولة فلة على حدا ثم تحديد الفرق بينهما. 
عرض التجربتين الأمريكية و الأوروبية يتيح للدول الأخرى و للشركات أيضا أن تستفيد منهما، من خلال التركيز على مزايا كل تجربة و تجنب سلبياتها. بدراسة و تحليل المقاربتين، يمكن للباحثين استخراج مقاربة نظرية أكثر فعالية في مجال المسؤولية الاجتماعية ل اللشركات. تكمن الأهمية العلمية للبحث في إثراء المكتبة العربية ببحوث تتعلق بهذا الموضوع الذي تقل فيه الدراسات العربية كثيرا على الرغم من أهميته.

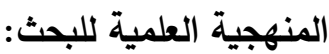

\section{1}

تم الاعتماد على المنهج الوصفي من خلال تحليل الظاهرة موضوع الدراسة باللجوء إلى المراجع الأساسية

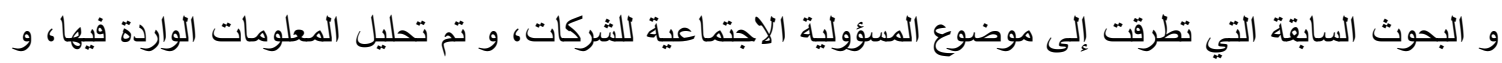

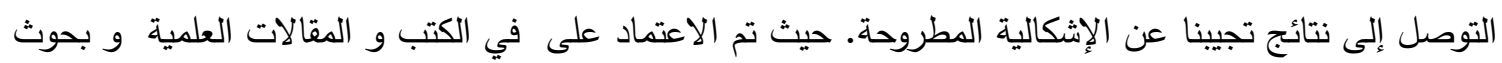

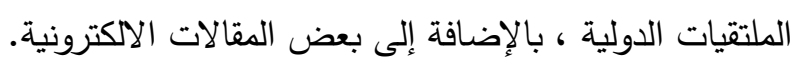

6.1

من أجل التعرض لمحاور الدراسة تم تقسيم البحث إلى العناصر التالية:

أولا: المقدمة، و نتعرض من خلالها للإطار المنهجي للدراسة

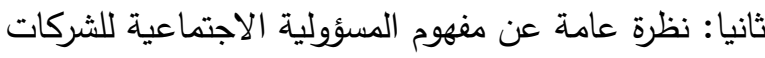

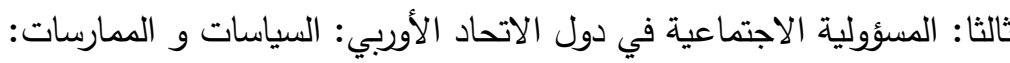

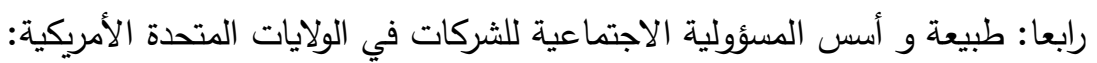

خامسا: أوجه الاختلاف بين المقاربات النظرية لمفهوم المسؤولية الاجتماعية للشركات بين دول الاتحاد

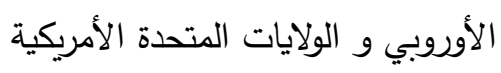

سادسا: الخاتمة، و فيها عرض لنتائج الدراسة و توصياتها.

2. نظرة عامة عن مفهوم المسؤولية الاجتماعية للشركات:

ظهر مفهوم المسؤولية الاجتماعية منذ حقبة زمنية بعيدة، حيث يمكن تتبع العديد من الأدلة على اهتمام القطاع الخاص في المجتمع والقضايا الاجتماعية لعدة عقود، و يرجح الباحثون أن نشأة المفهوم تعود إلى ثلاثينيات القرن

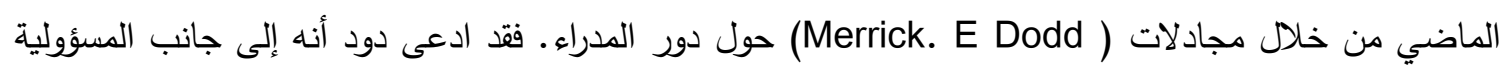

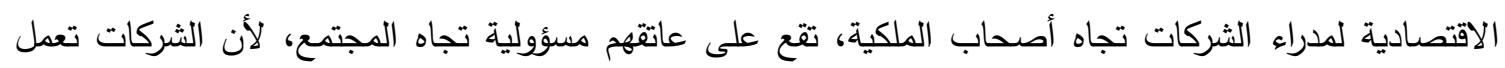
ضمن قانون يرخص لها ويشجعها على العمل بثكل أساسي خدمة للمجتمع وليس لتكديس الربح لأصحاب الثركات فقط غير أن الأدبيات الصريحة حول المسؤولية الاجتماعية هي إلى حد كبير نتاج سنوات الخمسينيات من القرن

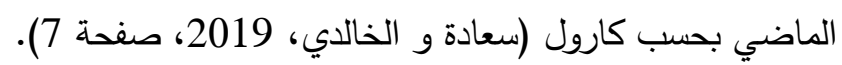
ونظرا لازدياد أهمية المسؤولية الاجتماعية فقد ظهر العديد من التعريفات التي حاولت تحديد التعريف الدقيق للمسؤولية الاجتماعية، نذكر منها: 
تعريف منظمة المقاييس العالمية ISO والتي عرفتها بأنها: مسؤولية المنظمة عن الآثار المترتبة لقراراتها وأنثطتها على المجتمع والبيئة عبر الثفافية والسلوك الأخلاقي المتناسق مع التتمية المستدامة ورفاه المجتمع فضلا عن الأخذ بعين الاعتبار توقعات المساهمين، فالمسؤولية الاجتماعية تعد التزاما على ولى ولى منظمة الأعمال تجاه المجتمع الذي تعمل فيه وذلك عن طريق المساهمة في مجموعة كبيرة من الأنشطة

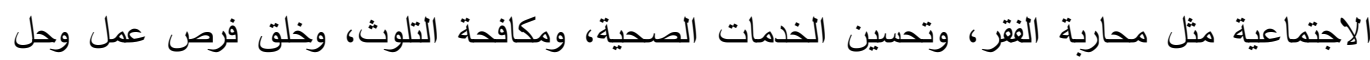
مشكلة الإسكان والمواصلات وغيرها.

و يعرفها البنك الدولي المسؤلية أنها التزام أصحاب النشاطات الاقتصادية بالمساهمة في التنمية

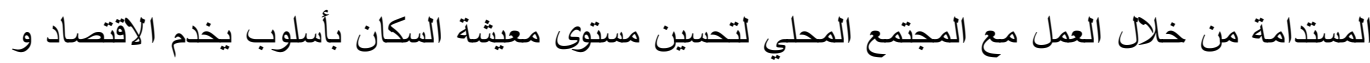

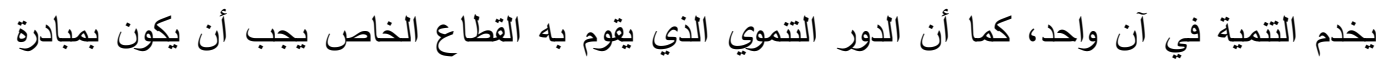

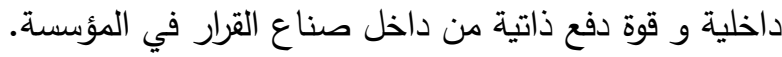

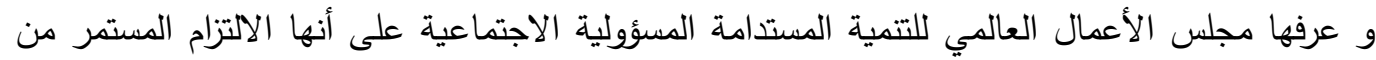

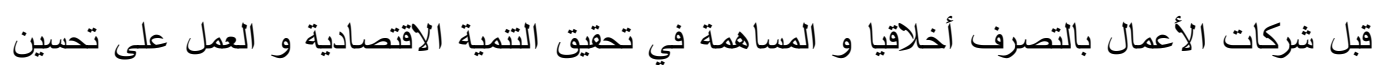
نوعية الظروف المعيشية للقوى العاملة و عائلاتهم و المجتمع ككل.

و من خلال التعاريف السابقة ، يمكن أن نخلص إلى التعريف التالي : المسؤولية الاجتماعية هي التزام منظمة

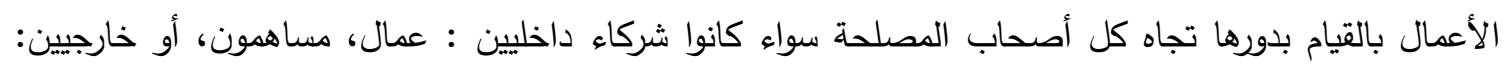

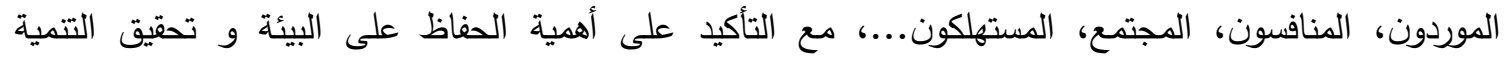
المستدامة و المساهمة بشكل فعال لحل مشكلات المجتمع و تجنب الإضرار به، و دون إهمالها للمكاسب المادية الماية

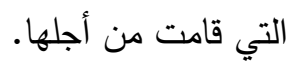

و من جهة أخرى فقد جاء (Carroll) بنقلة نوعية في توسيع مفهوم المسؤولية الاجتماعية، حيث ميز بين أربعة أبعاد رئيسية لهذا الدفهوم، البعد الأول هو الجانب الاقتصادي حيث تمارس المؤسسة أنثطة اقتصادية لتحقيق الكفاءة و الفعالية حيث تستخدم الموارد بشكل رشيد لتتنج سلع و خدمات بنوعية راقية و توزع العوائد بشكل عادل على عوامل الإنتاج المختلفة، بتحقيق ذلك تكون قد تحملت مسؤولية اقتصادية.

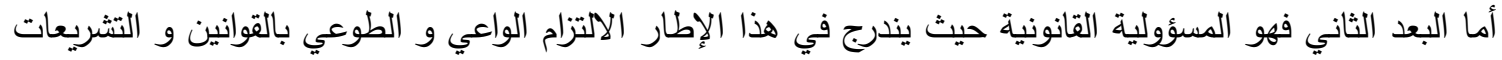

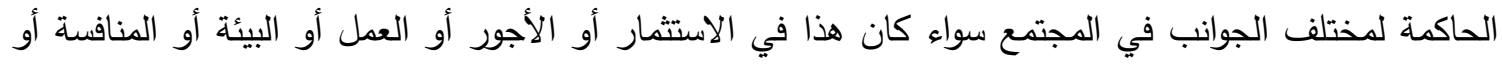
غيرها.

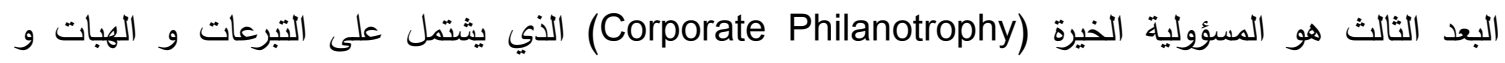
المساعدات الاجتماعية الخيرية التي تخدم الدجتمع و لا تهدف إلى الربح، كما قد تتنى المنظمة قضية أساسية من التئ قضايا المجتمع و تعمل على دعمها و متابعتها.

البعد الرابع هو المسؤولية الأخلاقية التي تراعي من خلاله المنظمة الجانب الأخلاقي في كل قراراتها و مسارها في

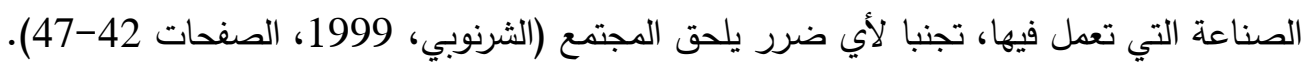


و جدير بالذكر أنه يجب أن تلتزم منظمات الأعمال بممارسة عدد من المسؤوليات الاجتماعية تجاه كل أصحاب

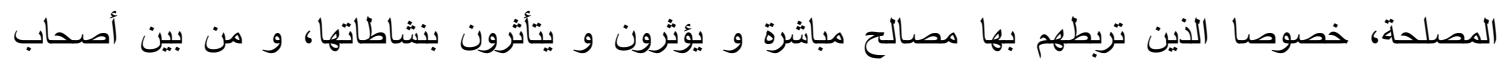

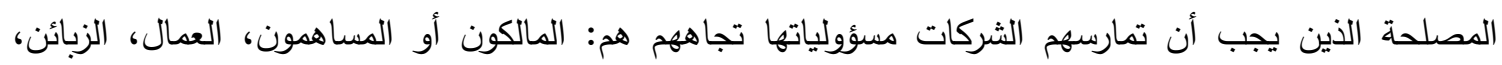

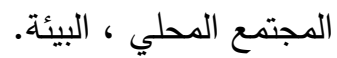

و أظهرت العددي من البحوث أن برامج الميؤولية الاجتماعية للشركات تزيد الأرباح في الأجل الطويل، وتجذب

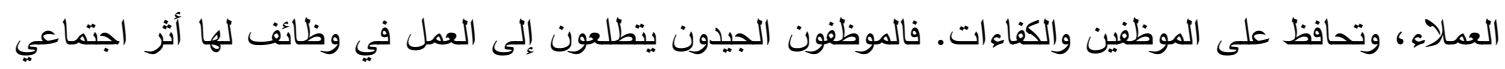
إيجابي في شركات تثاركهم نفس القيم، وتسع لآرائهم، وتقدر إنجازاتهم. ومن الآثار الإيجابية للمسؤولية

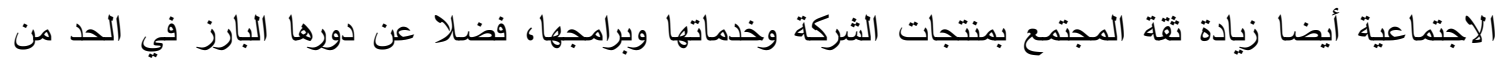
المخاطر التجارية والقانونية ورفع قدرتها على زيادة رأس المال.

3.المسؤولية الاجتماعية في دول الاتحاد الأوببي: السياسات و الممارسات:

تعرف المفوضية الأوروبية المسؤولية الاجتماعية للشركة على أنها مفهوم تدمج فيه الشركة بين الاهتمامات المجتمعية والبيئية في عملياتها التجارية وفي تعاملها مع أصحاب المصلحة على أساس طوعي. كما تدرك الثركة الثرونة بثكل متزايد أن السلوك المسئول يؤدي إلى النجاح في عالم اجتماعيا الأعمال، وربط البعض مفهوم المسؤولية

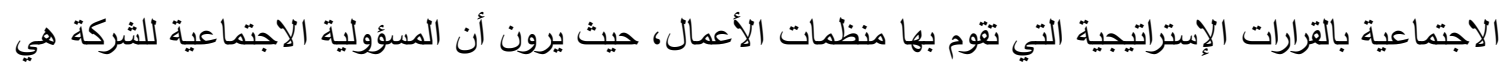

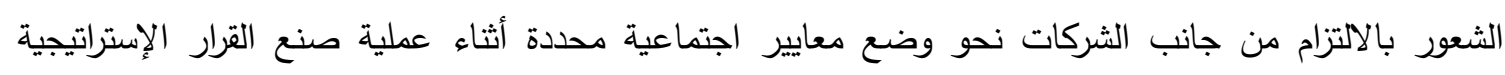
(النويقة، 2016، صفحة 120). و من خلال هذا التعريف يمكن أن نستخلص رؤية دول الاتحاد الأوروبي للمسؤولية الاجتماعية، حيث أن التعريف يركز على: المسؤولية الاجتماعية هي عمل طوعي و ليس إلزامي

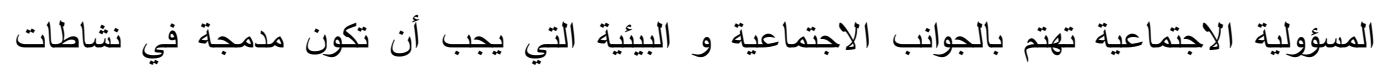
الشركة.

المسؤولية الاجتماعية تكون تجاه أصحاب المصالح المسؤولية الاجتماعية ترتبط بالقرارات الإستراتيجية للشركة التباهيه

و على المستوى الأوروبي، تمت الاستجابة للجدل المثار حول المسؤولية الاجتماعية للمنظمات في أواسط

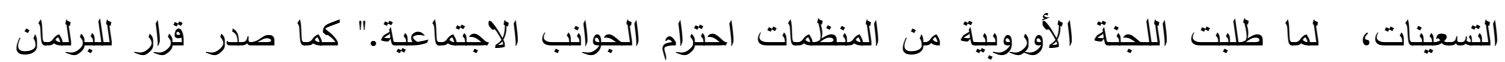

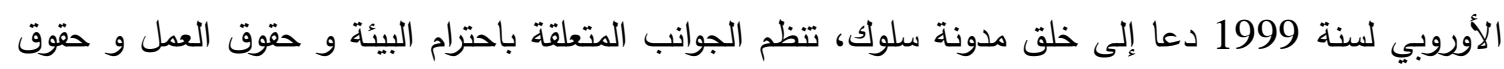
الإنسان من طرف المنظمات الأوروبية، من أجل دعم موضوع المسؤولية الاجتماعية كعنصر مهم في في أجندة

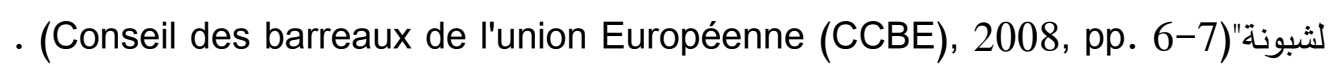
و صدرت سنة 2001 (الورقة الخضراء للمسؤولية الاجتماعية) من طرف اللجنة الأوروبية. و هو ما جعل

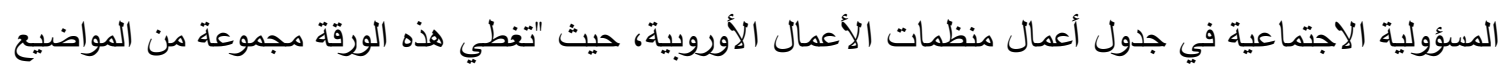
مثل إعادة هيكلة المنظمات من منظور المسؤولية الاجتماعية. إيجاد توازن بين العمل والحياة الثخصية. مدونات الاعليه

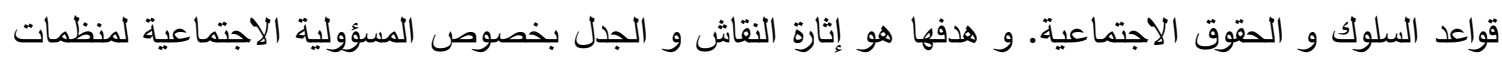


الأعمال الأوروبية، و حثها على إدماج الاهتمامات الاجتماعية و البيئية جنبا إلى جنب مع اهتماماتها الاقتصادية.

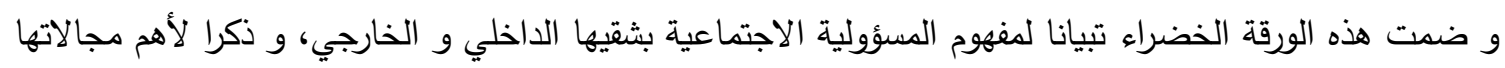
مع عرض نهج شامل لتبني المسؤولية الاجتماعية في المنظمات.

وشددت اللجنة الأوروبية من خلال هذه الورقة الخضراء على أهمية وجود "نهج شامل" للمسؤولية

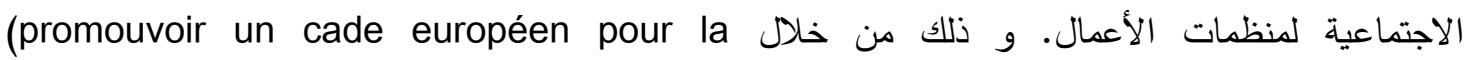
:esponsabilité sociale des entrepises, 2010)

• تطوير التدريب المناسب للموظفين لأهداف جديدة للمسؤولية الاجتماعية.

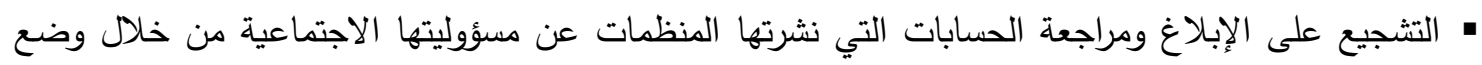

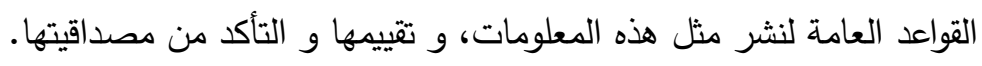
• ينبغي تشجيع الحوار مع موظفي المنظمة و إشراكهم في اتخاذ مختلف القرارات الإدارية. • تشجيع المعايير الأخلاقية استجابة لرغبات المستهلكين، حيث تقترح هذه الورقة آليات للتحقق من درجة الامتثال للقيم في عمليات الإنتاج. • زيادة الاستثمار المسئول اجتماعيا، حيث أن صناديق الاستثمار المسئول اجتماعيا يمكن أن تكون أداة جيدة لتعزيز المسؤولية الاجتماعية. • تظهر الورقة خضراء أهمية الحاجة إلى ضمان مزيد من التقارب بين المؤشرات التي وضعتها المؤسسات لتصنات والمعايير المستخدمة من قبل المحلين لتقييم الأداء الاجتماعي والبيئي. كما أن إقامة منتدى أوروبي متعدد الأطراف في سنة 2002 سمح بتبادل الخبرات حول المسؤولية الاجتماعية ووضع مبادئ مدونة السلوك، و الوصول إلى اتفاق حول طرق تقييم المسؤولية الاجتماعية.

و في سنة 2006 نشرت اللجنة الأوروبية دراسة بعنوان: "|إقامة شراكة من أجل النمو و التوظيف:جعل

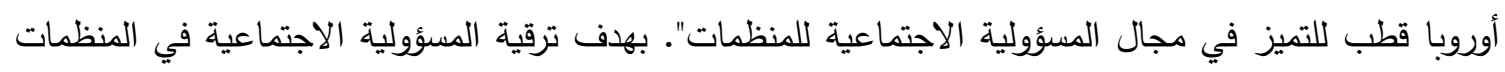
الأوروبية من خلال تأسيس تحالف يشكل الإطار السياسي العام للمبادرات الجديدة أو الموجودة سابقا في مجال

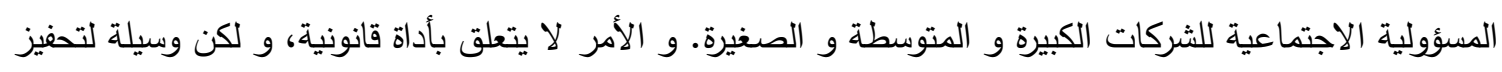

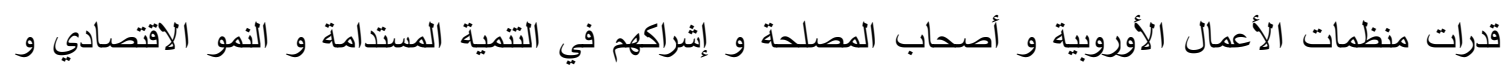
خلق مناصب الثغل.

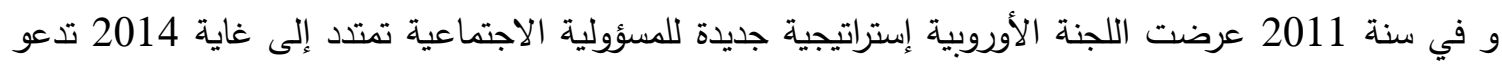

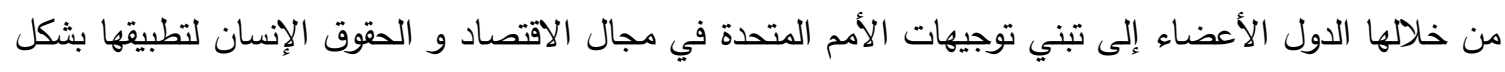

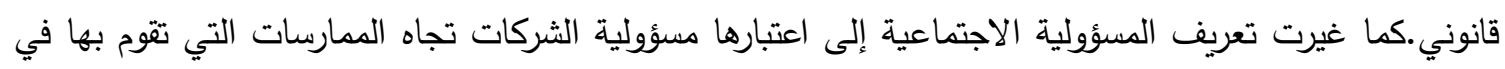
المجتمع.

و تتعكس السياسات الحكومية الأوروبية في مجال المسؤولية لاجتماعية من خلال دور أساسي و هو خلق تأطير

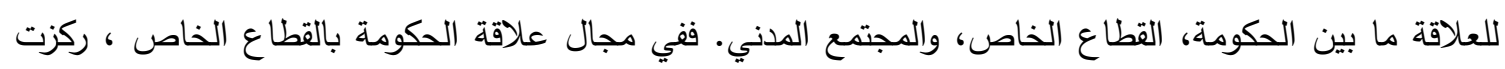

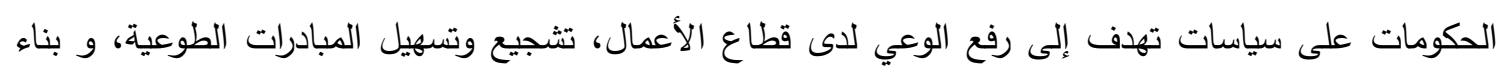

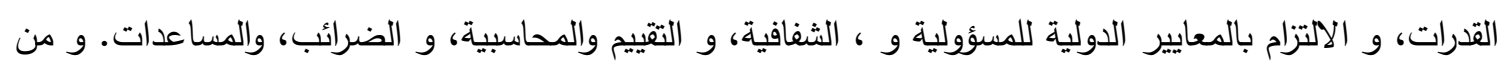


السياسات التي تم إتباعها لتحفيز المسؤولية، وربما يمكن أن نلتمس فيها جانب رقابي، إلزام الثركات بإصدار تقارير دورية للمسؤولية ، على أن تتضمن هذه التقارير إصدار معلومات كافية حول سياسات الثركة في مجال المسؤولية، تعرض فيها الثركة مدى التزامها بالقضايا الاجتماعية. تتبع حكومات دول الاتحاد الأوروبي عامة إستراتيجية أصحاب العلاقة المتعددين في تحديد سياسات الماتية المسؤولية

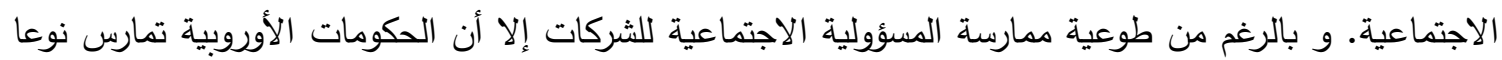

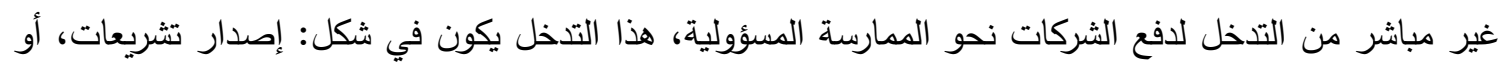

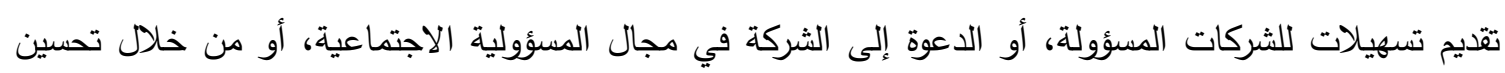

\section{4.طبيعة و أسس المسؤولية الاجتماعية للشركات في الولايات المتحدة الأمريكية:}

عرفت جمعية الإداريين الأمريكيين المسؤولية الاجتماعية بأنها استجابة إدارة الثركات العملية إلى التغير في

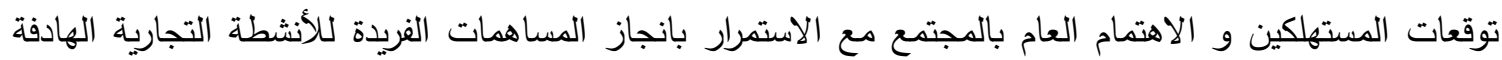

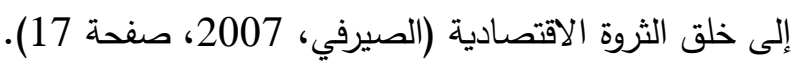
و قد بدأ اهتمام الولايات المتحدة الأمريكية بالمسؤولية الاجتماعية للمنظمات من خلال "المطالبة المتزايدة من قبل المبل

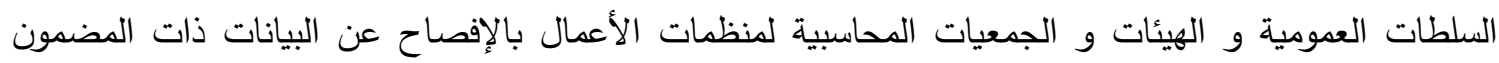

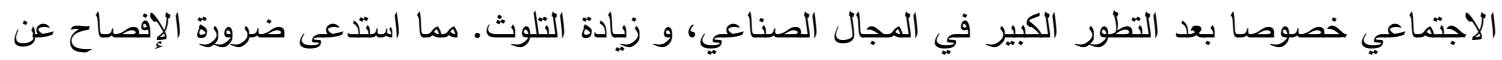
الأداء لمعرفة مدى قيام المنظمات بمسؤوليتها الاجتماعية. كما حدث توجه للمشاريع نحو أخذ التكاليف الاجتماعية

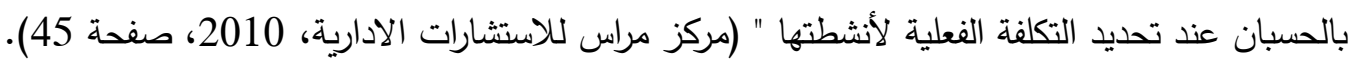
و يتفاوت تطبيق مفهوم المسؤولية الاجتماعية لرأس المال بين المنظمات في الولايات المتحدة الأمريكية بدرجة كبيرة. حيث تطبق بعض المنظمات الميؤولية الاجتماعية لسنوات طويلة جدا وصلت معها إلى درجة الاحترافية و

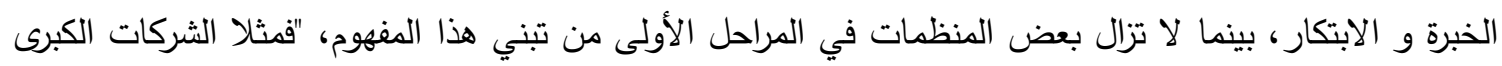
مثل : (Ford) و (General Motors) أصبحت تضع معايير خاصة بها لقياس مدى نجاحها في تطبيق برامج المسؤولية الاجتماعية، كما حققت برامج ناجحة للمسؤلية الاجتماعية محليا و عالميا، من خلال سلاسل القيمة

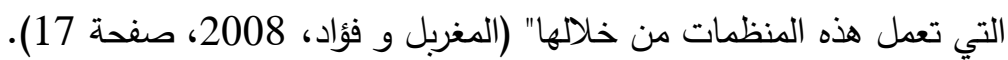

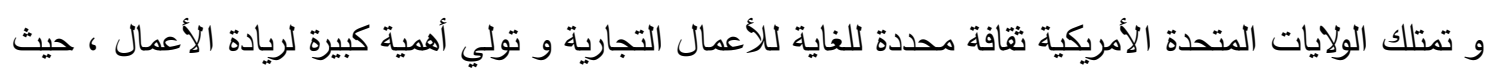

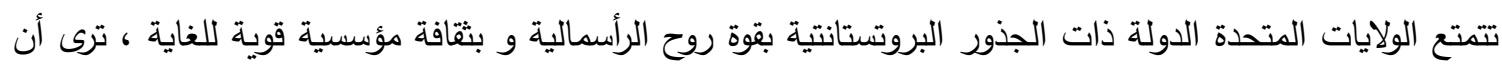

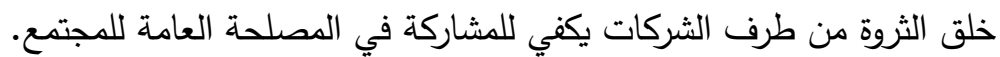
وبالتالي فإن الولايات المتحدة لديها مجموعة أخف من لوائح والتزامات المتعلقة بالمسؤولية الاجتماعية للشركات.

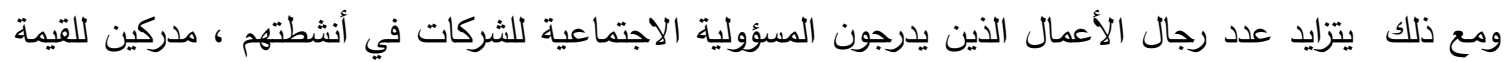

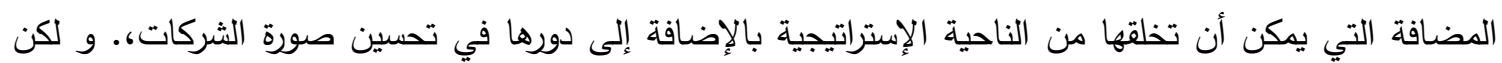
يبقى هذا النهج طوعي وفردي. فعلى سبيل المثال ، تحاول العديد من الثركات الأمريكية الانخراط في المجتمعات المحلية والتواصل مع المجتمعات التي تحتاج إلى المساعدة ومساعدتها من خلال المشاريع التطوعية والعمل الخيري، هذه الأخيرة تعد مشاريع مسؤولة اجتماعيا شائعة في الشركات الأمريكية الكبيرة. 
فمثلا تضمن شركة T\&AT أن تدفع لموظفيها يومًا واحدًا سنويًا حتى يتمكنوا من التطوع في منظمة من اختيارهم،

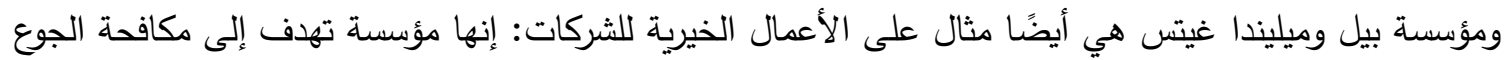

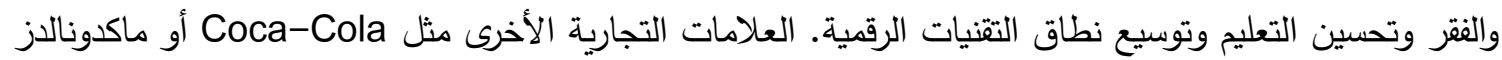
تمول الأحداث الخيرية أو الأحداث الرياضية مثل الألعاب الأولمبية. في الدول الأوروبية تتميز المسؤولية الاجتماعية للشركات بشكل أكبر بوجود توجيهات تتظيمية و تشريعات مثل: التزامات إعداد التقارير ، والبصمة الكربونية ، و غير ذلك، حيث تعتبر الحكومات الأوروبية مهندسا لرفاهية

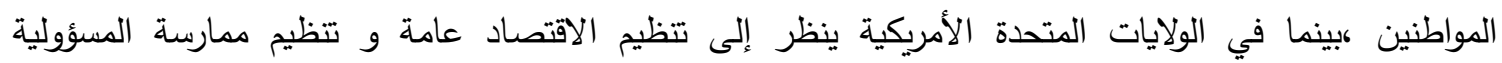
الاجتماعية على أنه تدخل غير مشروع في الأنشطة الاقتصادية و الحرية الخاصة للشركات و الأفراد.

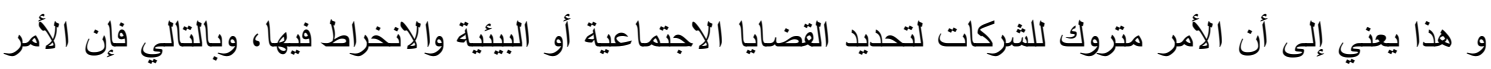
متروك لها لاختيار كيفية حلها. إذن يجب أن يكون لدى الشركات خيار يتعلق برغبتها في ممارسة المسؤولية الاجتماعية أولا بكل حرية و بطريقة ممارستها لهذه المسؤولية. و بالتالي فإن المسؤولية الاجتماعية للشركات في الولايات المتحدة هي بالأساس مسألة خاصة و ولهي هي أمر يتعلق

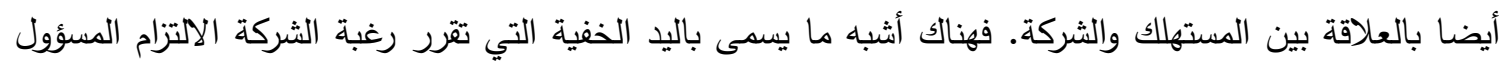

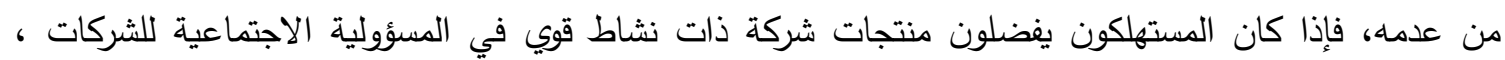
فسوف يزداد الطلب على سلع و خدمات هذه الثركة ، مما يمنح الشركات ميزة تتافسية. و تحصل بذلك على دافع

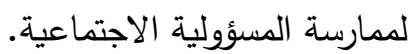
إذن يتميز المفهوم "المتحرر" للمسؤولية الاجتماعية للشركات بترك الحرية للثركات في اختيار و تطبيق إستراتيجية المسؤولية الاجتماعية من دون تعقيدات قانونية أو إجراءات تتظيمية كما هو معمول به في أوروبا و قد حققت

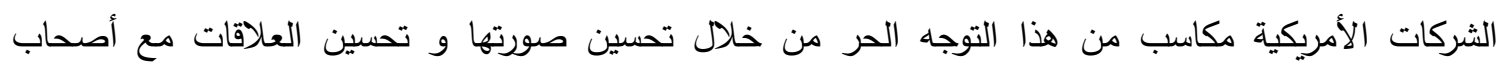

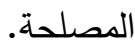

و لكن من ناحية أخرى، يطرح هذا مشكلة تحديد متطلبات المصلحة العامة. ففي الولايات المتحدة الأمريكية لا يوجد

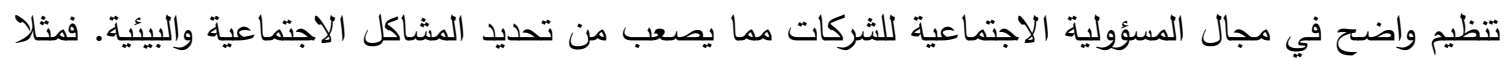

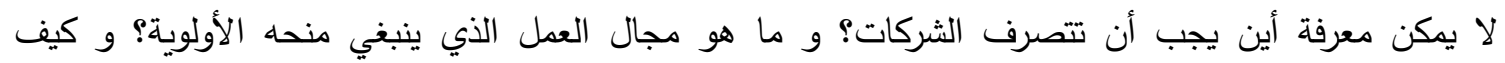
تتصرف؟ فالدعم و التوجيه الحكومي مفقود في الولايات المتحدة.مما أقلق الكثير من المتخصصين الأمريكيين في

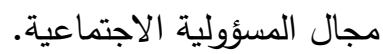
و على العكس من ذلك، ففي أوروبا تدفع العديد من القيود والحوافز القانونية الثركات للحد من بصمتها البيئية أو اعتماد ظروف عمل أفضل في الخارج أو تحسين دورها المسؤول عامة ، و بما أن هذا القيد غير موجود أو بالكاد موجود في الولايات المتحدة، فإن الشركات ليس لديها الدافع لتحسين ممارساتها خاصة إذا كان هذا لا يجلب لها ميزة تنافسية. فإلى جانب القوانين الأساسية القائمة بشأن التلوث أو التمييز العنصري أو التحرش الجنسي في العمل، لا يوجد

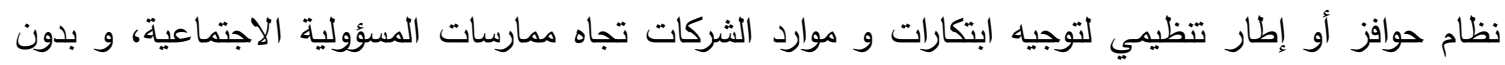
ضغوط من المستهلكين يمن للشركات بكل بساطة تجاهل المسؤولية الاجتماعية بالكامل في الولايات المتحدة لوناه

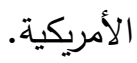


ولكن مع ظهور الاتجاهات الاستهلاكية المسؤولة، يبدو من الصعب بشكل متزايد الاستغناء عن المسؤولية الاجتماعية للشركات. لذلك من غير المستبعد أن تتجه الولايات المتحدة الأمريكية لتنظيم ممارسات المسؤولية

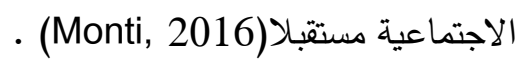

5.أوجه الاختلاف بين المقاربات النظرية لمفهوم المسؤولية الاجتماعية للشركات بين دول الاتحاد الأوروبي و الولايات المتحدة الأمريكية:

هناك العديد من الاختلافات المرتبطة برؤية كلا من دول الاتحاد الأوروبي و الولايات الأمريكية المتحدة لجوهر

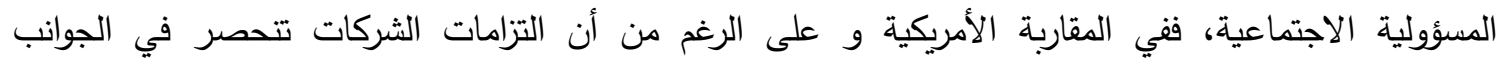

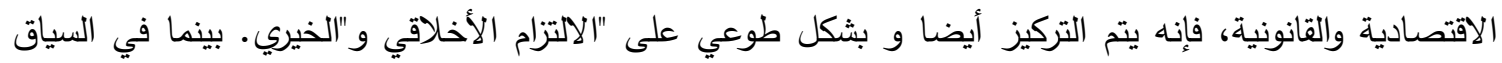

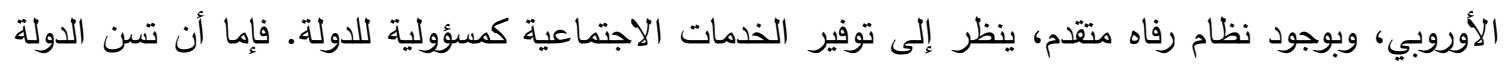
القوانين التي تضمن السلوك المسؤول من خلال برامج حكومية، أو أن تدعم الفئات المحرومة اجتماعيا واقتصاديا.

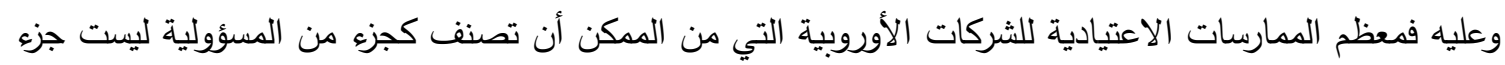
من السياسات المعلنة للشركات (مسؤولية اجتماعية ضمنية) ، إنما تتضمنها الأعراف وتقاليد العمل و الالتزام

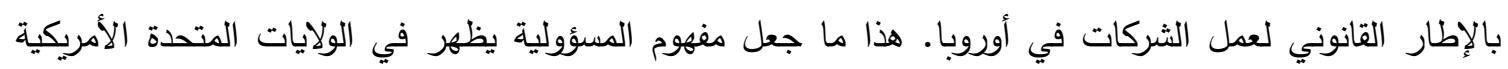

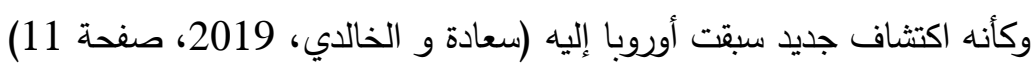
و هذا يظهر أن الإطار القانوني والنسيج المؤسساتي في أوروبا تضمن الكثير من القضايا التي ظهرت ضنمن إنهان

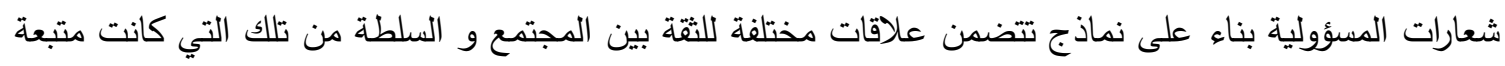
في الولايات المتحدة الأمريكية الأكثر لييرالية اقتصاديا ، فعلى سبيل المثال فيما يتعلق بالبعد الخيري للمسؤولية ،

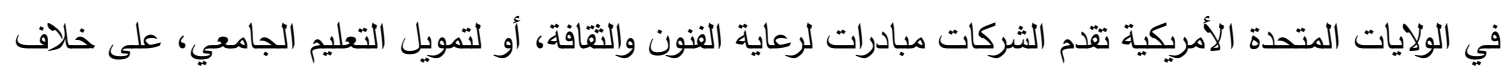

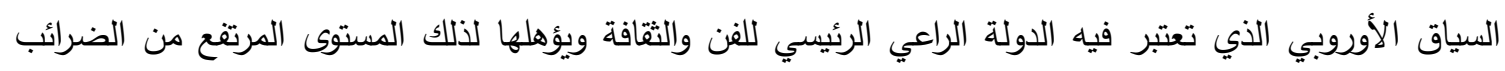
المفروضة على الشركات. فبرزت بشكل كبير التشريعات الأوروبية وتوجيهات النقابات الداعمة للفئات الاجتماعية المختلفة والضامنة لحقوقها مما شكل توجه الثركات نحو الممارسات التي تتعلق بالقضايا الاجتماعية والبيئية ومصلحة الاقتصاد الكلي. فالمسؤولية كسياسة طوعية لا تزال ظاهرة حديثة ومتتاثرة في السياق الأوروبي، بسبب الأشكال المختلفة تاريخيا من علاقات الثقة والسلطة لدول الرفاه الاجتماعي الأوروبية، على عكس النموذج الليبرالي كما في الولايات المتحدة

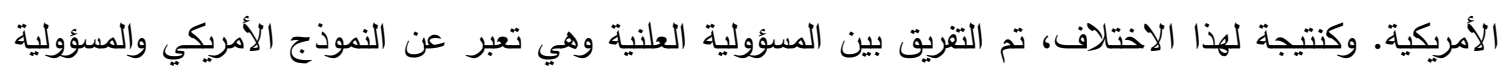

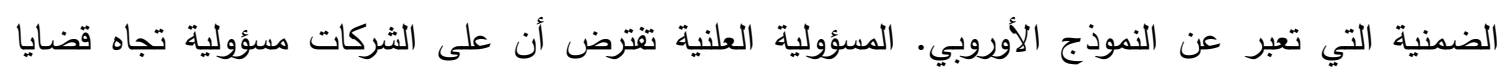

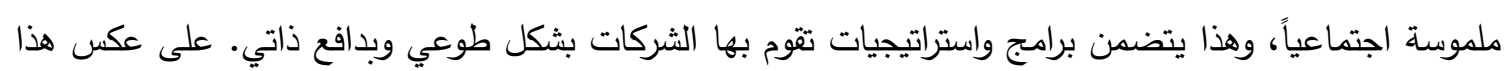
النموذج، نجد المسؤولية الضمنية ويعمل هذا الثكل سواء في المؤسسات الرسمية وغير الرسمية ويعبر عن التزام

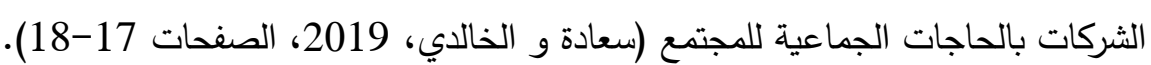
و خلال العقد الأخير تولت الحكومات الأوروبية تحفيز وقيادة المسؤلية الاجتماعية للشركات من خلال تبني

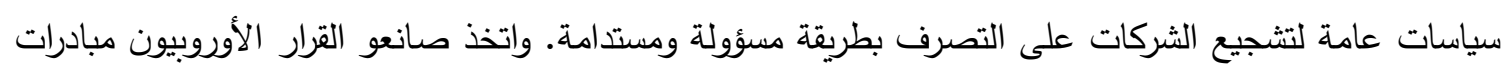
لتثجيع المسؤولية الاجتماعية للشركات على نطاق واسع، على عكس الولايات المتحدة الأمريكية حيث السياسات 
المخصصة لهذا المجال محدودة. كما أن "الثركات في أوروبا أكثر تقبلا للعمل مع الحكومة لتحسين ظروف العمال، و معتادة على العمل في بيئة منظمة. و بثكل عام هناك مجموعة من الخطوط العريضة المتعلقة بالمسؤولية الاجتماعية و التي تشكل محور خلاف

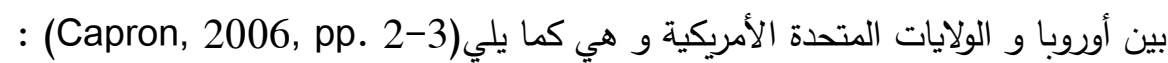

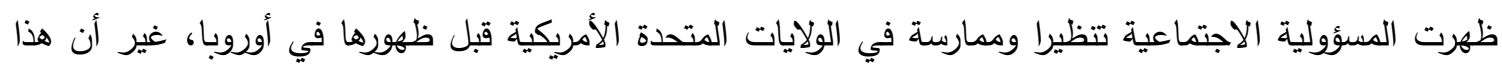

يكون صحيحا فقط عند الحيث عن المسؤولية الاجتماعية بشكلها الصريح.

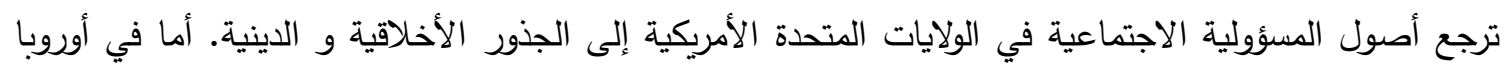

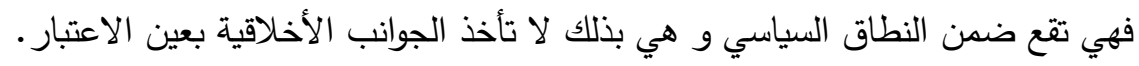

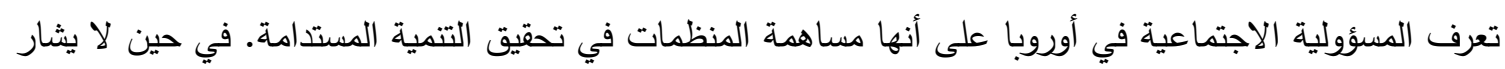

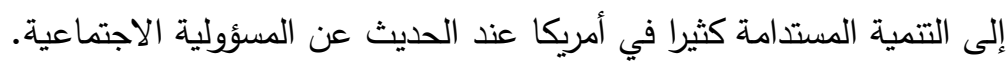

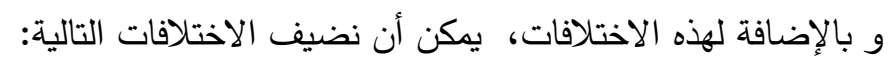

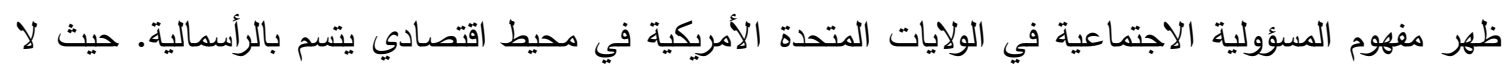

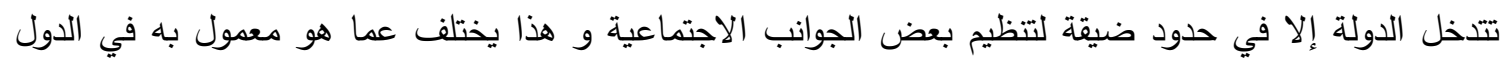
الأوروبية حيث تتذخل الدولة بثكل بارز في دعم و تثجيع برامج الدسؤولية الاجتماعية.

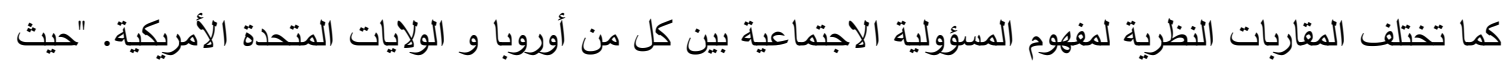

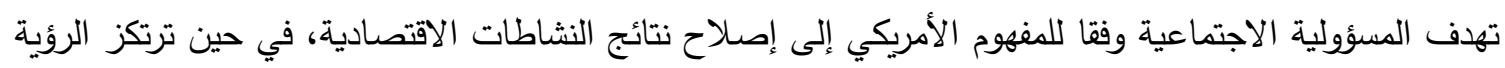

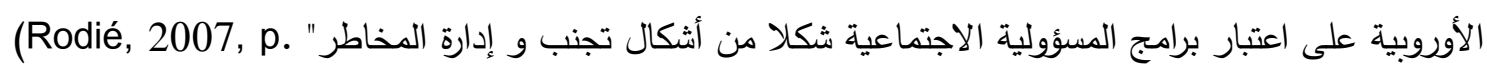

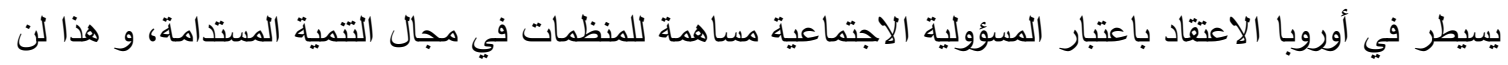

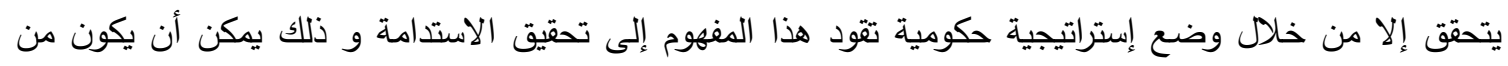

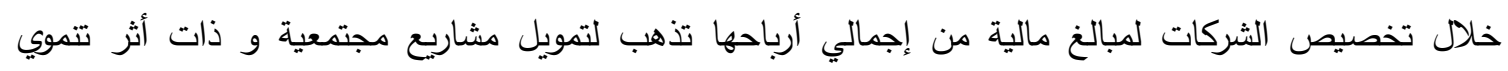

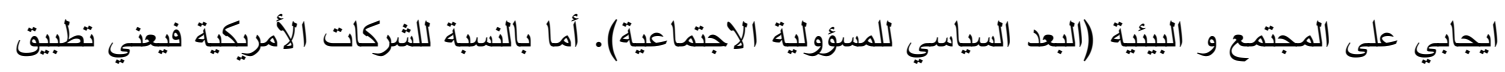

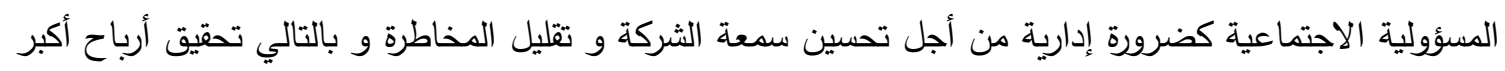

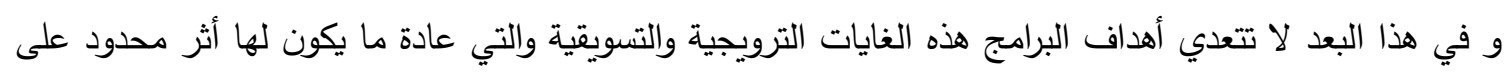
المجتمع (البعد الأدائي للمسؤولية الاجتماعية).

5.الخاتمة:

بالرغم من أن مفهوم المسؤولية الاجتماعية للشركات لم يعد موضوعا حديثا في مجال غدارة الأعمال، إلا أنه ما

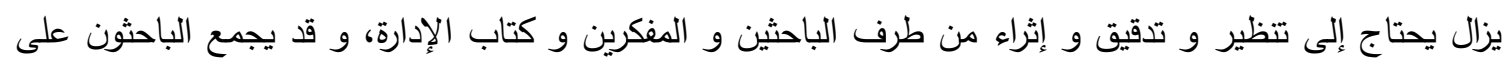

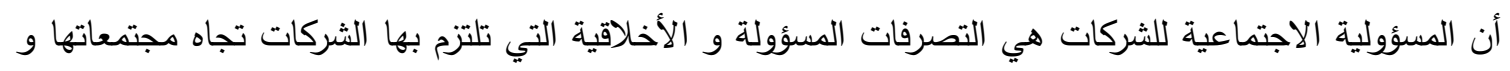

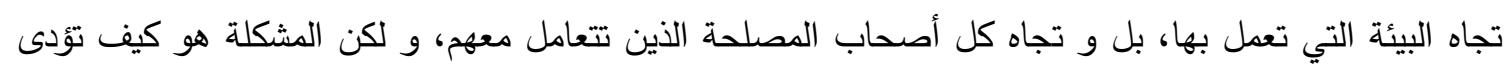
هذه المسؤولية الاجتماعية؟

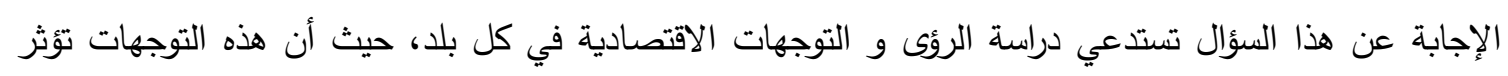

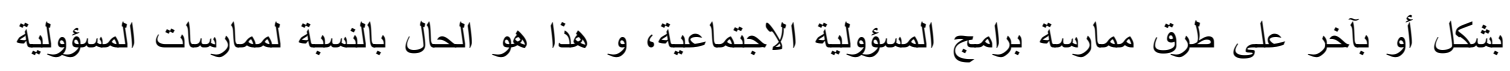


الاجتماعية في كل من الولايات المتحدة الأمريكية و الاتحاد الأوروبي.حيث تتأثر هذه الممارسات بخصائص

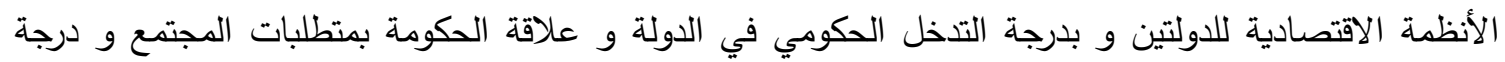
الحرية الممنوحة للشركات في مجال ممارسة العمل المجتمعي المسؤول. و في ختام هذه الدراسة يمكن أن نلخص أهم الاختلافات التي تميز الفرق بين المسؤولية الاجتماعية في الدول الأوروبية و المسؤولية الاجتماعية في الولايات المتحدة الأمريكية:

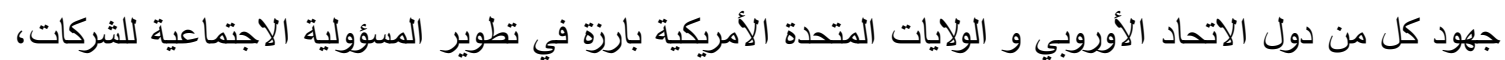
لكن لكل منهما طريقته الخاصة، ففي أوروبا تحرص على الحكومات على مرافقة الشركات في مسعاها المسؤول بشكل دائم و تتظيمي و تشريعي، أما في أمريكا فتترك الثركات لاختيار استراتيجياتها في مجال المسؤولية

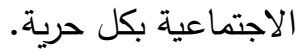
ترجع أصول المسؤولية الاجتماعية في الولايات المتحدة الأمريكية إلى الجذور الأخلاقية و الدينية التي تميزها. أما

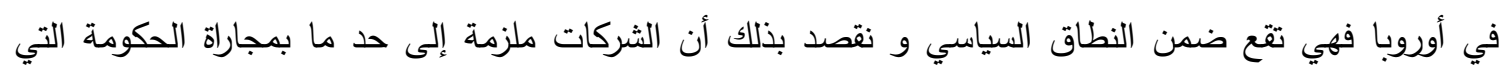
تفرض عليها الوفاء بمنطلبات المسؤولية الاجتماعية وفق أطر تتظيمية و تشريعية، إذن فالمسؤولية الاجتماعية في أوروبا لا تأخذ الجوانب الأخلاقية بعين الاعتبار . تمارس الشركات الأوروبية ما يسمى بالمسؤولية الاجتماعية الضمنية و التي نقصد بها أن برامج المسؤولية

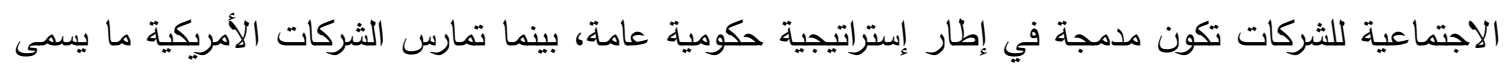

بالمسؤولية الاجتماعية العلنية، حيث أن الثركات هي المسؤولة بشكل مستقل عن برامج المسؤولية الاجتماعية.

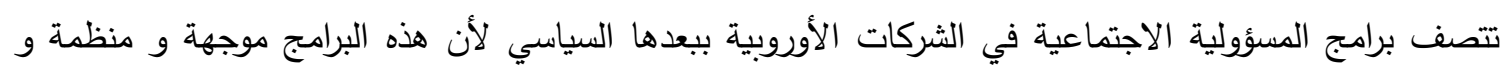

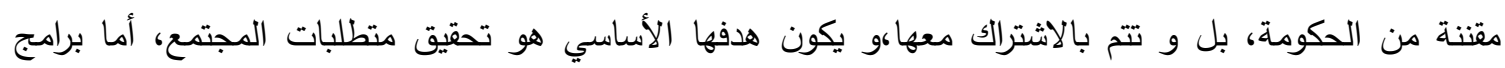

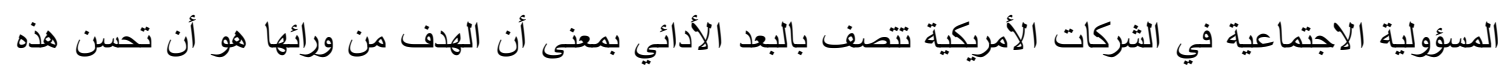
الثركات سمعتها و صورتها في ذهنية المتعاملين معها و بالتالي تكتسب ميزة تتافسية.

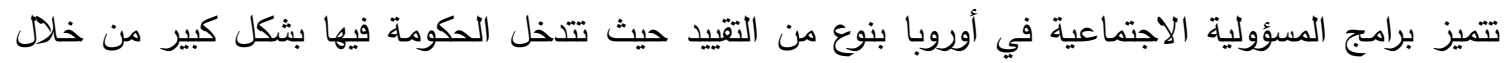

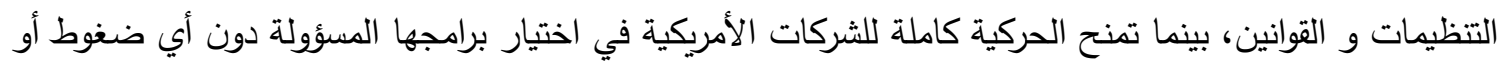
قوانين أو تعليمات (المسؤولية الاجتماعية المتحررة).

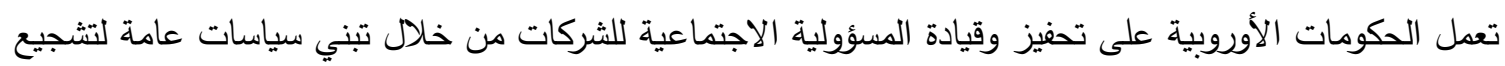
الثركات على التصرف بطريقة مسؤولة ومستدامة. واتخذ صانعو القرار الأوروبيون مبادرات لتشجيع المسؤولية

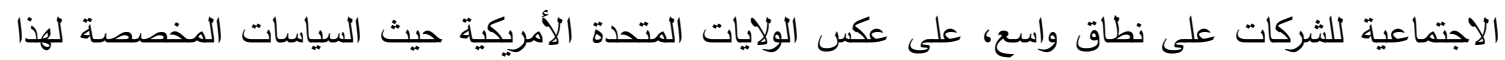

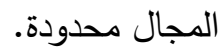

تهدف المسؤولية الاجتماعية وفقا للمفهوم الأمريكي إلى إصلاح نتائج النشاطات الاقتصادية، في حين ترتكز الرؤية

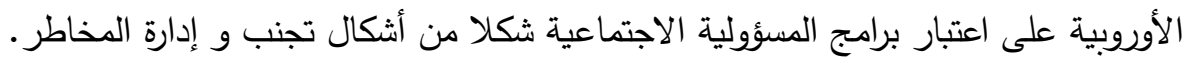

و في الأخير يمكن أن نعرض بعض التوصيات و المقترحات المتعلقة بالدراسة: تحتاج المقاربات النظرية للميؤولية الاجتماعية في دول الاتحاد الأوروبي و الؤلايات المتحدة الأمريكية إلى المزيد من البحث و الدراسة و التحليل من قبل الباحثين من أجل فهم أفضل و من أجل معرفة ايجابيات و سلبيات كل مقاربة. 
على الرغم من اختلاف المقاربتين، إلا أن الثركات الأوروبية و الأمريكية حقتت نجاحا كبيرا في مجال المبؤولية الاجتماعية للشركات، لذلك يمكن الاستفادة من مزايا كل مقاربة و تجنب السلبيات فيهما.

يعتبر درجة تدخل الدولة في سياسات و برامج المسؤولية الاجتماعية الحد الفاصل الذي يميز بين المقاربتين فبين

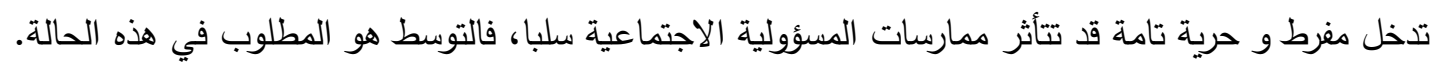

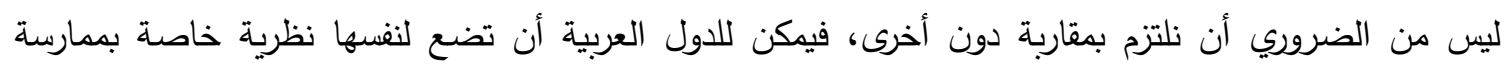
المسؤولية الاجتماعية، تتجنب من خلالها مساوئ المقاربتين و تستفيد من مزاياهما.

المغربل رن \& ,.فؤاد ,ي .(2008) .المسؤولية الاجتماعية لرأس المال في مصر :بعض التجارب الدولية .المركز

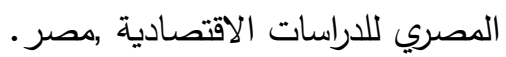

ايمان سعادة، و رجا الخالدي. (2019). واقع العسؤلية الاجتماعية للشركات الفلسطينية و سبل توجيهها نحو دعم التندية الاجتماعية. فلسطين: معهد أبحاث السياسات الاقتصادية الفلسطيني ماس).

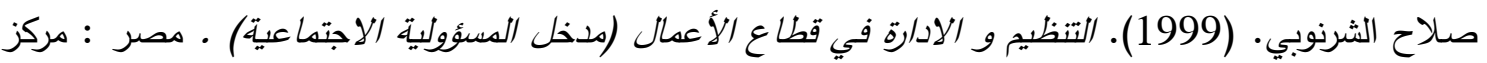

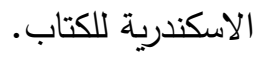

عطا الله بثير النويقة. (2016). أثر أخلاقيات الأعمال و المسؤولية الاجتماعية في تعزيز الميزة التنافسية في البنوك التجارية العاملة في منطقة مكة المكرمة. مجلة دراسات (العلوم الادارية) ، الدجلد 43 (العدد 1). محمد الصيرفي. (2007). المسؤولية الاجتماعية للادرة. الاسكندرية، مصر : الوفاء لدنيا الطباعة و النشر. مركز مراس للاستشارات الادارية. (2010). تحرير مفهوم المسؤولية الاجتماعية للشركات. سلسلة تطوير المسؤولية الاجتماعية للشركات، مجلس المسؤولية الاجتماعية بالرياض، الرياض.

\section{References}

Almugharbel, N., \& Fouad, Y. (2008). The social responsibility of capital in Egypt: some international experiences. The Egyptian Center for Economic Studies, Egypt. (in Arabic)

Atallah Bashir Al-Nuwaiqa. (2016). The impact of business ethics and social responsibility in enhancing skills in commercial banks operating in the Makkah region. Journal of Studies (Administrative Sciences), 43(1). (in Arabic)

Capron, M. (2006). Une vision européenne des différences USA/Europe continentale en matière de RSE :pourquoi la RSE en Europe est un objet politique et non pas éthique, le séminaie intenational de Montréal sue la RSE.

Conseil des barreaux de l'union Européenne (CCBE). (2008). la responsabilité des entreprises et le role de la pofession Européens. guide a l'attention des avorats Européens, Bruxelles.

Meraas Management Consulting Center. (2010). Liberalizing the concept of corporate social responsibility. Corporate Social Responsibility Development Series, Social Responsibility Council in Riyadh, Riyadh, KSA. (in Arabic) 
Monti, N. (2016). Comment les entreprises Américains conçoivent-elles la RSE. Retrieved 5 13, 2020, from Youmatter: https://youmatter.world/fr/rse-etats-unisusa-reglementation-differences-france/

Muhammad Al-Sayrafi. (2007). Management social responsibility. Alexandria, Egypt: Alwafa world of printing and publishing. (in Arabic)

Promouvoir un cade européen pour la esponsabilité sociale des entreprises. (2010). Consulté le 1 13, 2011, sur SENAT: www.senat.fr/ue/pac/E1776.html

Rodié, I. (2007). Responsabilité sociale des entreprises-le développement d'un cadre européen; mémoire présenté pour l'obtention du diplôme d'étude européennes, Genève.

Saadeh Iman , and Khalidi Raja. (2019). The reality of Palestinian corporate social responsibility and ways to direct it towards supporting social development. Palestine: The Palestinian Economic Research Institute (MAS). (in Arabic)

Salah Al-Sharnoubi. (1999). Organization and management in the business sector (introduction to social responsibility). Egypt: Alexandria Book Center. (in Arabic) 